\title{
Modelación hidrológica distribuida aplicada a la cuenca hidrográfica del río Júcar (España)
}

\author{
Distributed hydrology modeling applied to Jucar river basin (Spain)
}

\author{
Ramos Fernández Lía ${ }^{1}$; Félix Frances García
}

\begin{abstract}
Resumen
La lluvia en la cuenca del Júcar es un proceso complejo asociado a un grado de incertidumbre que se caracteriza por su enorme variabilidad temporal y espacial. En verano, encontramos lluvias intensas de corta duración, con picos aislados de valor muy alto, originadas por sistemas convectivos de mesoescala SCM típicos del Mediterráneo; en invierno, los valores más altos se concentran en la parte alta de la cuenca, originados por la cadena de montañas del sistema ibérico. Ante esto, el objetivo del presente trabajo es obtener la respuesta hidrológica ocasionada por episodios de lluvia, teniendo en cuenta los diferentes procesos físicos involucrados y empleando la modelación distribuida, aplicándola a la cuenca del río Júcar, para un periodo de análisis que abarca del $1^{\circ}$ de enero del 2000 al 31 de octubre del 2009. La información obtenida del estudio de la modelación hidrológica tuvo óptimos resultados con índices de Nash-Sutcliffe de 0,87 (calibración), 0,81 (validación temporal) y 0,51-0,62 (validación espacio-temporal) y por tanto podría proporcionar información que serviría de ayuda en diversas aplicaciones hidrológicas, gestión del agua y del medio ambiente en la cuenca del río Júcar. Con ello, se fortalecería la capacidad de gestión de los recursos hídricos, se mejoraría la predicción del clima y los desastres naturales y se ofrecería rigor científico para ayudar a tomar decisiones informadas.
\end{abstract}

Palabras clave: modelación hidrológica; modelo distribuido; río Júcar.

\begin{abstract}
The rainfall in the Jucar Basin is a complex process that is associated with a degree of uncertainty which is characterized by its temporal and spatial variability. In summer time, intense rain of short duration, with isolated peaks of very high value, which are caused by mesoscale convective systems MCS typical of the Mediterranean. During winter, the highest values are concentrated in the upper part of the basin, these is caused by the chain of mountains of the Iberian system. Given this, the aim of this work is to obtain the hydrological response caused by rain events, applying a distributed model at the Jucar Basin for the period that goes from January 1, 2000 to October 31, 2009. The information obtained from the study of the hydrologic modeling had excellent results with Nash-Sutcliffe index of 0,87 (calibration), 0,81 (temporal validation) and 0,51 to 0,62 (spatial and temporal validation) therefore it could provide information which will help in various hydrological applications, water and environmental management in the Jucar Basin. This could strengthen the management capacity of water resources, improve weather and natural disasters forecasting and could also provide scientific rigor that will help to make informed decisions.
\end{abstract}

Keywords: hydrological modeling; distributed model; Jucar river.

\section{Introducción}

La variabilidad espacial de las precipitaciones y las propiedades de la cuenca tienen impactos significativos en la respuesta hidrológica de estas. Caracterizar y modelar la relación entre la distribución espacial de la lluvia, las características de la cuenca y la generación de escorrentía ha sido objeto de numerosos estudios durante más de dos décadas. Debido a que no se dispone de un marco matemático que explique satisfactoriamente el comportamiento del flujo dentro de una cuenca, la modelación hidrológica distribuida ha sido el punto de interés de la comunidad científica en los últimos años.
Las ventajas que presenta la modelación distribuida con respecto a la tradicional modelación agregada consisten, fundamentalmente, en la mejor representación de la variabilidad espacial de los fenómenos involucrados en los procesos hidrológicos. Adicionalmente, la modelación distribuida ha surgido para lograr un mejor entendimiento de los procesos en los niveles de la cuenca y la parcela. Aunque en ocasiones requiere de información detallada de la zona de estudio, este tipo de información se encuentra, día a día, más a disposición del público gracias al desarrollo de los ordenadores, la cartografía digital, los sistemas de información geográfica y las mediciones con sensores

1. Departamento de Recursos Hídricos. Universidad Nacional Agraria La Molina, Lima, Perú. Email: liarf@lamolina.edu.pe

2. Institute of Water and Environmental Engineering. Technical University of Valencia, España. Email: ffrances@hma.upv.es 
remotos (Ekhakbaz et al., 2009). Para la estimación y la simulación de las crecidas, se ha encontrado que los procesos físicos involucrados más importantes son la lluvia y la escorrentía superficial, por lo tanto se ha generalizado en la literatura el término lluvia-escorrentía. Dentro de estos modelos, se encuentra el modelo TETIS, que es la herramienta utilizada en el presente trabajo de investigación, desarrollada por el Departamento de Ingeniería Hidráulica y Medio Ambiente de la Universidad Politécnica de Valencia. Por lo anteriormente expuesto, el objetivo de este trabajo es obtener la respuesta hidrológica ocasionada por episodios de lluvia, teniendo en cuenta los diferentes procesos físicos involucrados y empleando la modelación distribuida de tipo conceptual TETIS aplicada a la cuenca del río Júcar.

\section{Materiales y métodos}

\section{Modelo TETIS}

TETIS es un modelo hidrológico distribuido conceptual con parámetros físicamente basados que simulan los principales procesos del ciclo hidrológico. La producción de escorrentía se modela utilizando siete tanques conectados en cada celda de modelación, describiendo las interacciones atmósfera-vegetación-suelo-acuífero (Fig. 1). En el estudio no se usó el tanque de manto de nieve (To) como tampoco el tanque de intercepción de la vegetación (T6). Los flujos verticales de agua entre cada tanque representan los procesos hidrológicos de precipitación (lluvia o nieve, X6), evaporación directa (Y6), precipitación efectiva (X1), evapotranspiración potencial (Y1), infiltración gravitacional (X3), percolación (X4) y pérdidas subterráneas (X5), mientras que los flujos horizontales representan los procesos hidrológicos de escorrentía directa (Y2), interflujo (Y3) y flujo base (Y4). El movimiento horizontal del flujo ocurre separado en dos fases; en la primera, la escorrentía directa, interflujo y flujo base están definidos por una malla de tanques de tres capas conectados entre sí, en donde el movimiento del agua es hacia el correspondiente tanque aguas abajo, siguiendo las direcciones del flujo propuestas por el modelo de elevación

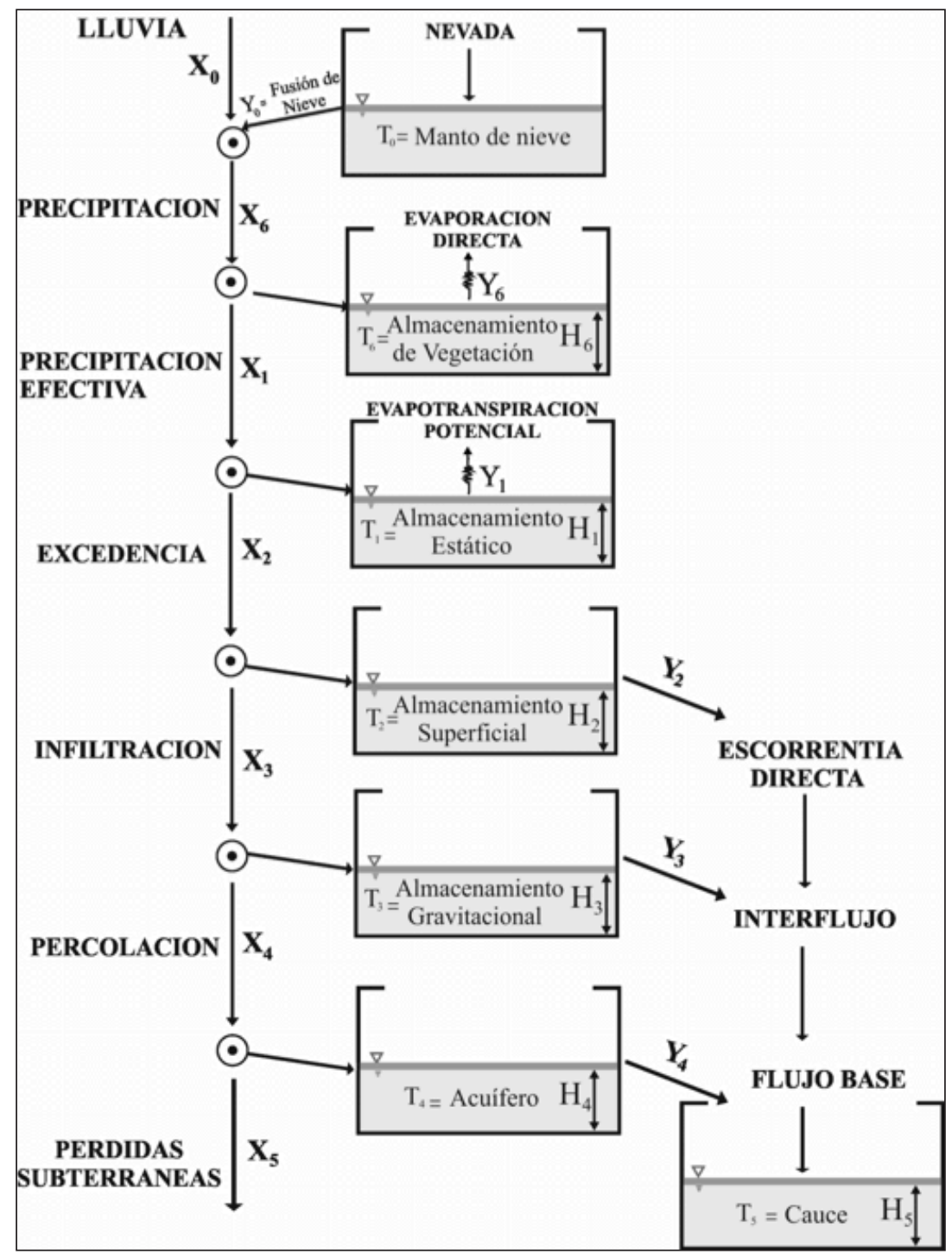

Figura 1. Esquema conceptual vertical de la producción de escorrentía en el modelo TETIS en cada celda (las variables se describen en el texto) 
digital (MED), hasta alcanzar la red principal de drenaje; en la segunda fase se da el movimiento del flujo sobre la red de cauces. La propagación en cauces es gobernada por la onda cinemática teniendo en cuenta las características geomorfológicas de la red de cauces, en lo que se denomina onda cinemática geomorfológica (Frances et al., 2007; Vélez y Frances, 2008; Vélez et al., 2009).

\section{Parámetros del modelo TETIS}

Vélez (2001) indica que el modelo TETIS establece la forma de la sección de cada uno de los cauces que conforman la red a partir de relaciones de geometría hidráulica, las cuales fueron propuestas por Leopold y Madock (1953), quienes relacionaron las dimensiones de la sección de flujo, como profundidad (y), ancho (w) y velocidad (v) con el caudal en el cauce $(\mathrm{Q})$ mediante ecuaciones de tipo potencial: (y $=\mathrm{b} . \mathrm{Q} \beta),(\mathrm{w}=\mathrm{c} \cdot \mathrm{Q} \alpha)$ y $(\mathrm{v}=\mathrm{p} \cdot \mathrm{Q} \lambda)$. El modelo emplea nueve parámetros geomorfológicos del cauce $(\mathrm{k}, \alpha, \mathrm{Cd}, \mathrm{Cn}, \phi, \alpha 1$, $\alpha 2, \theta$ y $\xi$ ) para caracterizar la geometría y la resistencia al flujo en la red de cauces. Esta información es necesaria para transitar el flujo superficial en la red de cauces empleando el método de la onda cinemática. Los parámetros son establecidos mediante relaciones algebraicas de tipo potencial entre algunas características geométricas y/o hidráulicas del cauce representativo de cada celda y una variable asociada a un caudal "a sección llena"; a su vez, este caudal se relaciona con el área acumulada hasta la celda. Las relaciones geomorfológicas que intervienen en el cálculo son: área acumulada de la cuenca y caudal a sección llena $(\Lambda=\kappa \mathrm{Qb} \phi)$; ancho de la sección transversal a sección llena y caudal a sección llena $(\mathrm{wb}=\alpha \mathrm{Qb} \alpha 1)$; ancho de la sección transversal y caudal de estación ( $\mathrm{w}=$ Q 2 2); diámetro del sedimento, pendiente y profundidad del flujo $(\mathrm{d}=\mathrm{Cd}$ (y so) $\theta)$ y coeficiente de rugosidad y diámetro del sedimento $(\mathrm{n}=\mathrm{Cn} \mathrm{d} \xi)$ (Frances et al., 2007). Los coeficientes y los exponentes de las ecuaciones anteriores pueden estimarse utilizando regresiones lineales, a partir de un número pequeño de secciones transversales medidas en campo, para cada región geomorfológica homogénea. Los parámetros efectivos permiten simular procesos hidrológicos con ecuaciones de escala pequeña, pero aplicando una escala grande, de tal modo que las salidas del modelo con ambas escalas mantengan una similitud. Estos parámetros permiten simplificar la complejidad geométrica de los esquemas de modelación y, en consecuencia, reducir el requerimiento de datos y el esfuerzo computacional. Sin embargo, en modelos distribuidos la calibración de un elevado número de parámetros en cada celda resulta un problema. Frances et al. (2007) proponen una estructura separada del parámetro efectivo, que se detalla en la Fig. 2. La ventaja principal de esta estructura es que, en la fase de calibración, se reduce notablemente el número de variables que deben ser ajustadas, siendo solo necesario calibrar nueve factores correctores.

El modelo tiene un potente algoritmo de optimización Shuffled Complex Evolution de la Universidad de Arizona (SCE-UA) para calibración automática de sus parámetros y valores iniciales de las variables de estado, que permite de forma global una corrección rápida y ágil de los diferentes procesos representados (Vélez y Frances, 2009) . Para más información sobre el modelo TETIS se puede consultar a Vélez et al., 2002; Vélez y Frances, 2005; Frances et al., 2007.

Zona de estudio.

El área de estudio es la cuenca del río Júcar, con un área de $2.143,4 \mathrm{~km}^{2}$ (Fig.8) que incluye parte de las provincias de Albacete, Cuenca y Valencia, en España. El río Júcar es uno de los de mayor valor económico en España debido a su uso muy intenso: canales para riego, abastecimiento de agua potable, embalses para generar energía hidroeléctrica,

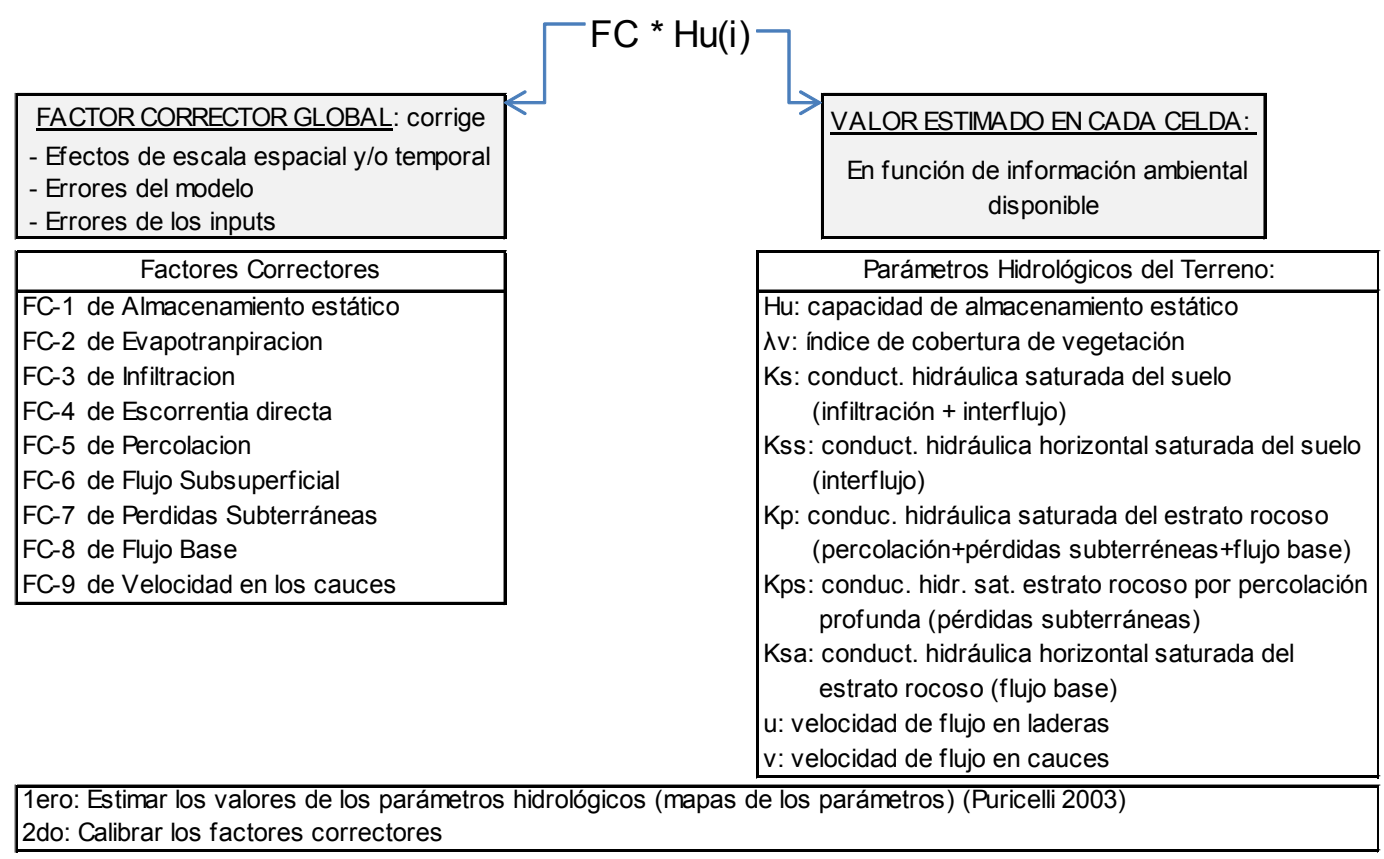

Figura 2. Estructura separada del parámetro efectivo en el modelo TETIS 
navegación fluvial, pesca fluvial, pesca deportiva, turismo, lo cual intensifica la competencia por el recurso hídrico. En general, tiene un clima mediterráneo con veranos cálidos y secos e inviernos suaves. La temperatura media anual varía de $9{ }^{\circ} \mathrm{C}$, en las zonas montañosas, a $18^{\circ} \mathrm{C}$, en la costa. El sistema hidrológico se caracteriza por lluvias de muy alta intensidad y corta duración de origen convectivo debido al fenómeno llamado "gota fría". El entorno de la cuenca puede definirse como una zona semiárida o incluso árida.

Dado que el modelo hidrológico TETIS es distribuido, la información cartográfica y parámetros hidrológicos de la cuenca deben estar distribuidos espacialmente, por tanto se empleó información cartográfica de la cuenca del Júcar (DIHMA, 2002) con tamaño de pixel 500 x 500 m que requirió una actualización y pre-procesamiento en ArcGIS para generar los mapas en formato que se ajusten al modelo hidrológico: modelo de elevación digital (MED), celdas acumuladas, direcciones de flujo, pendiente del terreno, velocidad en ladera y parámetros hidrológicos del terreno. Para los parámetros hidrológicos de la cuenca se empleó información del DIHMA (2002), que requirió una actualización y pre-procesamiento en ArcGIS para generar los mapas en formato que se ajusten al modelo hidrológico TETIS: capacidad de almacenamiento estático $(\mathrm{Hu})$, conductividad hidráulica saturada del suelo $(\mathrm{Ks})$, conductividad hidráulica saturada del estrato rocoso $(\mathrm{Kp})$ y conductividad hidráulica saturada del estrato rocoso por percolación (Kps). Cuanta más información se tenga, se podrá usar un tamaño de celda (pixel) más pequeño, pero en contrapartida se empleará un tiempo computacional mayor en la modelación. Por otro lado, cuanto más pequeño sea el tamaño de celda, se podría hacer una diferenciación entre cárcavas y cauces; además, esto se vería reflejado en los parámetros geomorfológicos.

La información de parámetros geomorfológicos del cauce del río Júcar, se obtuvo del estudio de la cuenca del Tajo (DIHMA, 2001), estudio en el que se detallan

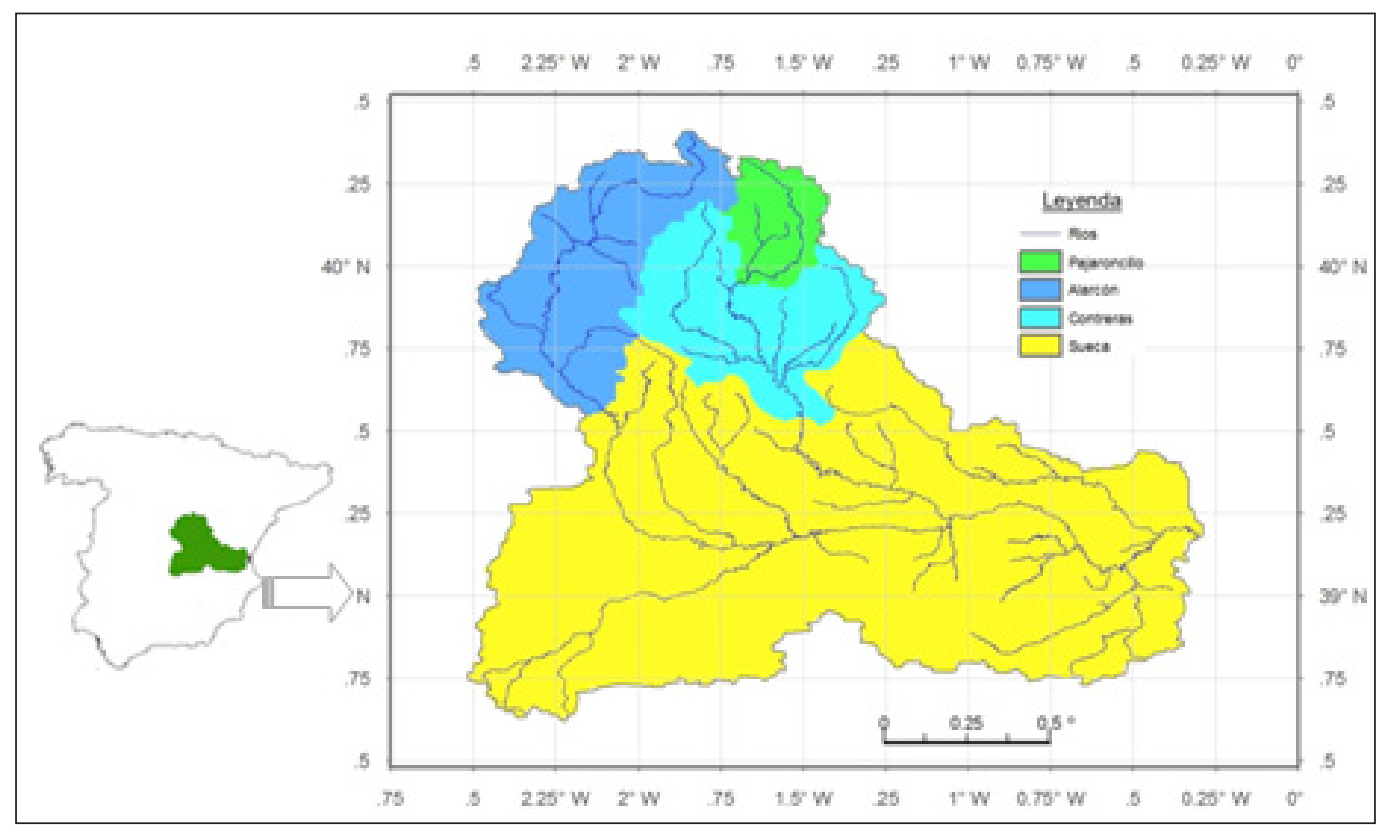

Figura 3. Cuenca hidrográfica del río Júcar

Tabla 1. Parámetros geomorfológicos del cauce del río Júcar. Elaborado con información de DIHMA (2001)

\begin{tabular}{|l|c|c|c|}
\hline \multicolumn{1}{|c|}{ Relaciones Geomorfológicas de Tipo Potencial } & $\mathrm{Y}=$ Coeficiente $\mathrm{X}^{\text {Exponente }}$ & \multicolumn{2}{|c|}{ B: Cuenca Media } \\
\cline { 3 - 4 } & $\Lambda=\kappa^{2} \mathrm{Q}_{\mathrm{b}}{ }^{\phi}$ & 1,2968 & 0,7571 \\
\hline $\begin{array}{l}\text { Área acumulada de la cuenca y caudal a sección } \\
\text { llena }\end{array}$ & $\mathrm{wb}=\alpha \mathrm{Q}_{\mathrm{b}}{ }^{\alpha 1}$ & 3,1301 & 0,5088 \\
\hline $\begin{array}{l}\text { Ancho de la sección transversal a sección llena y el } \\
\text { caudal a sección llena }\end{array}$ & $\mathrm{w}=\mathrm{Q}^{\alpha 2}$ & 0,3253 \\
\hline Ancho de la sección transversal y el caudal & $\mathrm{d}=\mathrm{C}_{\mathrm{d}}\left(\mathrm{y} \mathrm{s}_{\mathrm{o}}\right)^{\theta}$ & 6,5156 & 0,6548 \\
\hline $\begin{array}{l}\text { Diámetro del sedimento, la pendiente y la } \\
\text { profundidad del flujo }\end{array}$ & $\mathrm{n}=\mathrm{c}_{\mathrm{n}} \mathrm{d}^{\xi}$ & 0,0470 & 0,1667 \\
\hline $\begin{array}{l}\text { Coeficiente de rugosidad y el diámetro del } \\
\text { sedimento }\end{array}$ & \multicolumn{2}{|c|}{0} \\
\hline
\end{tabular}


cinco zonas geomorfológicas (zona de alta montaña, cuenca media, cuenca baja, zona de cabecera y cauce del río), eligiéndose para la cuenca del Júcar, por semejanza de zonas morfológicas, la subzona $\mathrm{B}$, cuyos valores de coeficiente y exponente se detallan en la Tabla 1. Como estos parámetros geomorfológicos influyen en la estimación de los parámetros de producción de escorrentía en la cuenca, la incertidumbre que se genera se soluciona con la calibración del factor corrector relacionado con la velocidad de ladera (FC9).

El índice de cobertura vegetal $(\lambda)$ mensual para la modelación de la evapotranspiración se obtiene según el tipo de cobertura vegetal, valores que se detallan en la Fig. 4.

La información de series temporales de lluvia ha sido facilitada por la Confederación Hidrográfica del Júcar (CHJ) y la Agencia Española de Meteorología (AEMET) con una densidad resultante de aproximadamente 1 pluviómetro por cada $116 \mathrm{~km}^{2}$. Esta densidad aumenta significativamente a 1 pluviómetro aproximadamente por cada $46 \mathrm{~km}^{2}$, en la parte baja de la cuenca, en que hay un mayor número de pluviómetros instalados cerca de la franja costera, que es donde se producen los eventos lluviosos más importantes. En la Fig. 5 se aprecia la distribución de las estaciones pluviométricas del SAIH y AEMET.

Además, se disponía de caudales aforados, información de embalses (volúmenes, caudales de entrada, caudales de salida) y temperatura. La información de aforos y embalses se recolectó a través del software SAIH Win proporcionado por la Confederación Hidrográfica del Júcar. El embalse



Figura 4. Variación mensual del índice de cobertura vegetal $(\lambda)$ en la cuenca del río Júcar. Elaborado con información del DIHMA, 2002

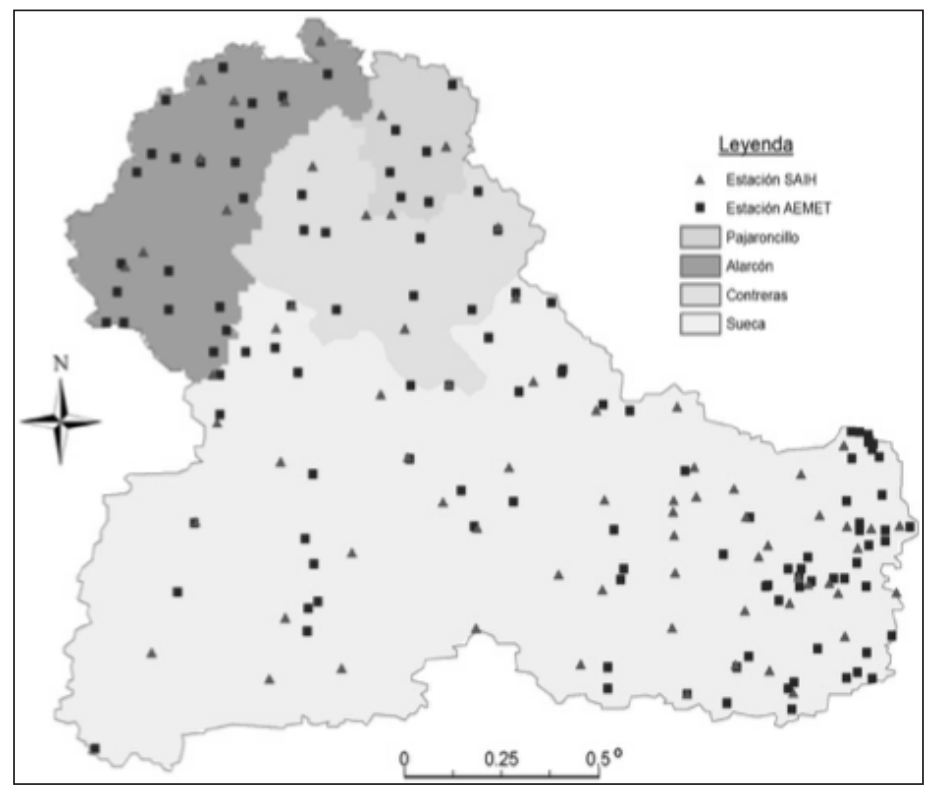

Figura 5. Estaciones pluviométricas SAIH y AEMET en la cuenca del río Júcar 
Alarcón recibe aguas del trasvase Tajo-Segura a la altura de Belmontejo y devuelve estas aguas a la altura de Picazo. Es por ello que en la modelación, fue necesario restar las aguas del Tajo-Segura al caudal de ingreso del embalse. Se requirió un arduo trabajo de análisis y posterior verificación de todos los registros, además de un pre procesamiento y codificación para ajustarse al tipo de formato compatible con el modelo TETIS.

Se disponía de información termométrica en 44 estaciones del AEMET, a partir de la cual se determinó la evapotranspiración de referencia (ETo) con la ecuación de Hargreaves (FAO, 2006): . Siendo Ro, la radiación extraterrestre tabulada que depende del mes y la latitud de la estación termométrica. Previamente, se calibró la ecuación de Hargreaves con valores de Penman-Monteith disponibles en las estaciones: "Las Tiesas" (disponible en http://www.itap.es/ITAP-Meteorologia/3infohistorica/ InfoHisto3.as) y "Requena Cerrito", "Bolbaite" y "Villanueva-Castellón" (disponibles en http://estaciones. ivia.es/).

Para la provincia de Albacete, se calibró con la estación "Las Tiesas" del Instituto Técnico Agronómico Provincial S.A. Se obtuvo un coeficiente de correlación alto $\left(\mathrm{R}^{2}=\right.$ $0,8617)$ y una constante de calibración de 0,0023679 que se empleó en el cálculo de la ETo en las estaciones termométricas de la provincia de Albacete y Cuenca.

Para la provincia de Valencia, se calibró con las estaciones Requena Cerrito, Bolbaite y Villanueva-Castellón, estaciones del Instituto Valenciano de Investigaciones Agraria-IVIA, escogiéndose la constante de calibración de la estación Requena Cerrito, que dio una mejor ajuste $\left(\mathrm{R}^{2}\right.$ $=0,8964)$. Entonces, la constante 0,0020617 se empleó en el cálculo de la ETo en las estaciones termométricas de la provincia de Valencia. Un resumen de las calibraciones realizadas se detalla en la Tabla 2.

Por ello, se obtuvo la ETo diaria desde el $1^{\circ}$ de marzo del 2000 al 31 de octubre del 2009, para todas las estaciones termométricas del AEMET, en la cuenca del Júcar.

\section{Metodología}

Preparación de información input.

Información espacial de la cuenca en formato ASCII que permitirán al modelo representar las características geomorfológicas y relaciones topológicas en la red de drenaje: MED, mapas de celdas acumuladas, mapa de direcciones de flujo, mapa de pendiente del terreno, velocidad en ladera.
Parámetros geomorfológicos del cauce según zonas geomorfológicas que ingresan como exponentes en ecuaciones geomorfológicas de tipo potencial (Frances et al., 2007).

Parámetros hidrológicos a escala de celda de la cuenca en formato ASCII: obtenida a partir de información ambiental disponible: capacidad de almacenamiento estático $(\mathrm{Hu})$, conductividad hidráulica saturada del suelo (Ks), conductividad hidráulica saturada del estrato rocoso $(\mathrm{Kp})$, conductividad hidráulica saturada del estrato rocoso por percolación (Kps), conductividad hidráulica horizontal saturada del suelo (Kss), conductividad hidráulica horizontal saturada del estrato rocoso (Ksa). Si no se dispone información de Kss, Ksa y Kps, una buena aproximación es: $\mathrm{Kss}=\mathrm{Ks}, \mathrm{Ksa}=\mathrm{Kp}$ y $\mathrm{Kps}=0,1 \mathrm{Kp}$. La estructura separada de los parámetros en TETIS permite luego la calibración de las distintas conductividades hidráulicas a través de cinco factores correctores diferentes por cada conductividad.

Índice de cobertura vegetal $(\lambda)$ que permite evaluar la variabilidad en el ciclo anual de la ETo mediante valores mensuales de $\lambda$ que representan el comportamiento del ciclo vegetativo de la cobertura vegetal.

Archivo input en formato CEDEX (Fig. 5) con información de intervalo temporal en días, fecha del evento, período de simulación en intervalos, series incompletas o fallos con código "-1", variables observadas que son input del modelo con código de inicio (lluvias en mm: $P$, caudales en $\mathrm{m} 3 / \mathrm{s}$ : Q; evapotranspiración en mm/día: E).

Calibración y validación.

La metodología de estimación de los parámetros hidrológicos involucra una serie de incertidumbres debido a la cantidad, calidad y grado de definición espacial de la información básica empleada; por esta razón, y con el propósito de obtener por medio del modelo los mejores resultados posibles, es necesario realizar un proceso de calibración o ajuste de dichos parámetros.

Por ejemplo, los parámetros $\mathrm{Hu}, \lambda$, Ks, Kp, Kss, expresan numéricamente las características más importantes de la cuenca; sin embargo, debido a que existen errores en las variables de entrada, errores propios del modelo y errores derivados de los efectos de escala, en TETIS se utilizan factores correctores (FC) para estimar los valores de los parámetros efectivos:

Factores Correctores: los que afectan directamente a la producción de escorrentía son: FC1 (corrige

Tabla 2. Resumen de Calibración de Hargreaves con Penman-Monteithcoma

\begin{tabular}{|l|c|c|c|c|c|c|c|}
\hline \multirow{2}{*}{ Estación } & \multirow{2}{*}{ Provincia } & Longitud & \multirow{2}{*}{ Oeste } & Latitud Norte & Altitud \\
msnm & & & $\begin{array}{c}\text { Error } \\
\text { Medio }\end{array}$ & R2 & Constante (C) \\
\hline Las Tiesas & Albacete & $2^{\circ} 5^{\prime} 10^{\prime}$ & $39^{\circ} 14^{\prime} 30^{\prime}$ & 695 & 0,782 & 0,8617 & 0,0023679 \\
\hline Requena Cerrito & Valencia & $1^{\circ} 6^{\prime} 00^{\prime}$ & $39^{\circ} 29^{\prime} 00^{\prime}$ & 692 & 0,407 & 0,8964 & 0,0020617 \\
\hline Bolbaite & Valencia & $0^{\circ} 41^{\prime} 20^{\prime \prime}$ & $39^{\circ} 4^{\prime} 13^{\prime \prime}$ & 269 & 0,383 & 0,8900 & 0,0020366 \\
\hline Villanueva-Castellón & Valencia & $0^{\circ} 31^{\prime} 22^{\prime \prime}$ & $39^{\circ} 4^{\prime} 0^{\prime \prime}$ & 58 & 0,465 & 0,8820 & 0,0020617 \\
\hline
\end{tabular}




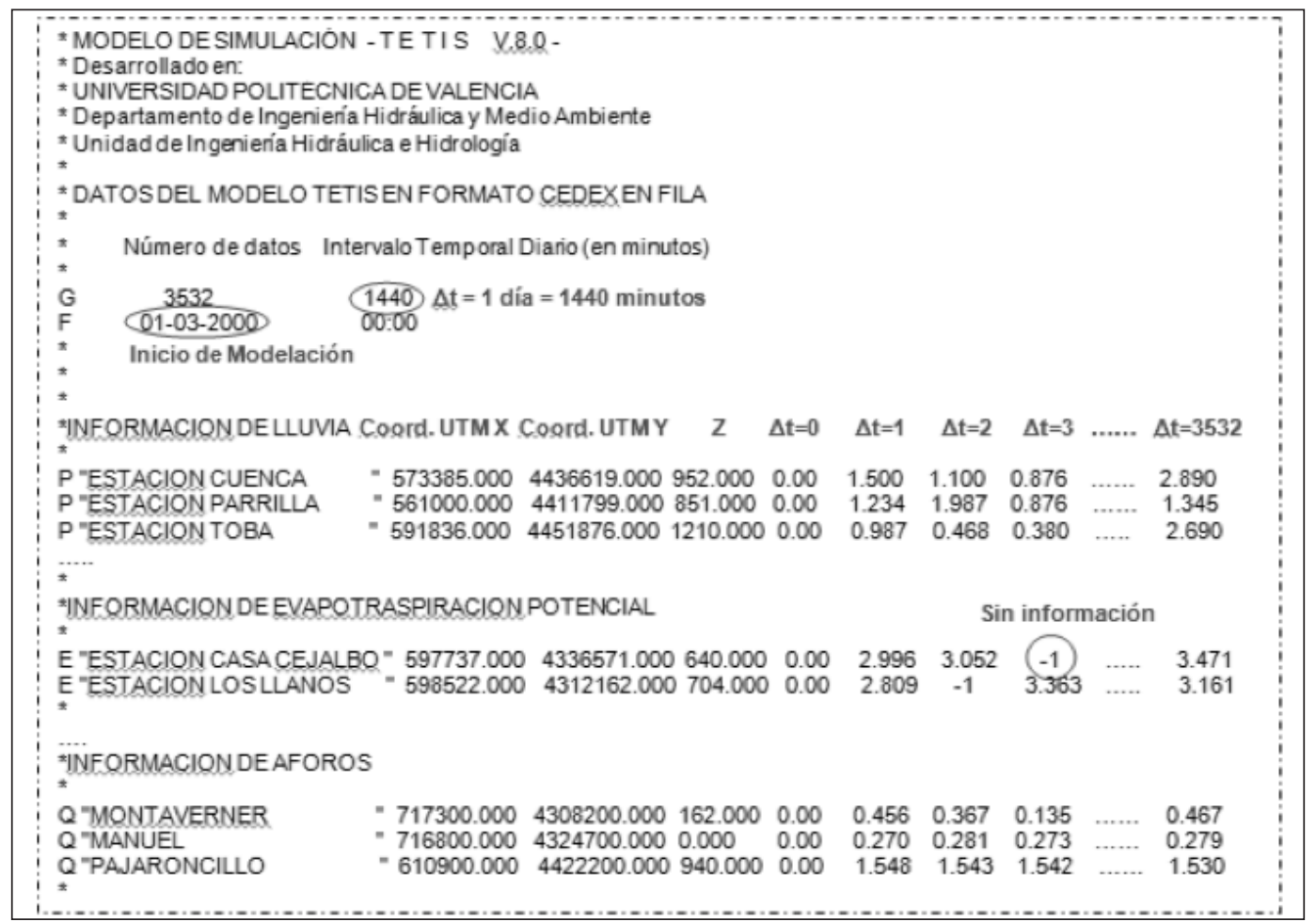

Figura 5. Formato de archivo input del modelo TETIS: archivo 01030000.e00 con inicio de modelación el $1^{\circ}$ de marzo del 2000

el almacenamiento estático), FC2 (corrige la evapotranspiración), FC3 (corrige la infiltración), FC4 (corrige la escorrentía directa), FC5 (corrige la percolación), FC6 (corrige el interflujo), FC7 (corrige las pérdidas subterráneas). Adicionalmente, para la traslación de la onda cinemática geomorfológica, se emplea un factor corrector para la velocidad de la onda FC9.

Calibración manual: el éxito depende esencialmente de la experiencia del modelador y de su conocimiento e interacción con el modelo. Además, la calibración siempre es subjetiva y puede consumir mucho tiempo (Eckhardt y Arnold, 2001). Se recomienda primero ajustar el flujo base, luego ajustar el porcentaje de error en el volumen que es más sensible a las condiciones iniciales de humedad en el tanque estático, acuífero y cauce; seguidamente, dejar los caudales picos para calibración automática.

Calibración Automática: los principales problemas que afectan a los algoritmos automáticos son (Duan et al., 1992): múltiples regiones de atracción, donde el óptimo encontrado depende del valor inicial; zonas de igual valor de la función objetivo con diferentes parámetros; tiempo computacional elevado.

Se recomienda ajustar los caudales picos con calibración automática y con función objetivo al Índice de Nash, que es más sensible a estos caudales. Es necesario realizar calibración manual antes que calibración automática ya que de lo contrario habrá un elevado número de iteraciones realizadas por el algoritmo SCE UA para converger a la solución, lo cual tiene repercusión directa en el tiempo de cómputo de la optimización.

Validación: con los factores correctores obtenidos en la calibración, se realizará la validación temporal y espacio- temporal; esta se ejecuta a escala temporal, si los registros con los que se pretende validar son obtenidos en la misma estación, pero en diferente fecha, y a escala espacial, si los registros a validar pertenecen a estaciones distintas, pero fueron registradas en el mismo intervalo temporal.

\section{Resultados y discusión}

La exacta precipitación no se conoce, sino que su valor se mide con un error. De hecho, la calidad y la precisión de los datos es un problema en la modelación hidrológica, por tanto, existe un grado de incertidumbre asociado. Por ello, el tipo de precipitación es más importante que la elección del modelo hidrológico en términos de producir simulaciones hidrológicas más robustas. Por otro lado, la metodología de estimación de los parámetros involucra una serie de incertidumbres debidas principalmente a la cantidad, calidad y grado de definición espacial de la información básica empleada. Ante esto, la ventaja del modelo TETIS es que se pueden corregir en gran medida los errores señalados, calibrando los factores correctores del modelo.

La calibración del modelo se comenzó realizando previamente su calentamiento con la finalidad de obtener las condiciones de humedad previas del sistema y el comportamiento del acuífero al inicio del periodo de calibración. La condición inicial, al comienzo del período de calentamiento, se obtuvo mediante ajuste manual, análisis de sensibilidad y comprobando, en todo momento, la estacionalidad de las variables de estado en el período de calibración. En la mayoría de las cuencas naturales, las pérdidas son una parte muy pequeña del agua que circula 
a través de la cuenca y, por lo tanto, se puede asumir que estas pérdidas son cero, lo cual se puede hacer ajustando el factor corrector FC7 $=0$ y así la totalidad del agua que percola pasa al almacenamiento subterráneo. La calibración final del modelo a la salida de la subcuenca Pajaroncillo, en el período de julio 2002 a julio 2003, generó resultados óptimos con factores correctores cuyos valores se detallan en la Tabla 3. Se observa un significativo interflujo y valores bajos de flujo base, lo cual concuerda con una conductividad hidráulica reducida, ya que se trata, fundamentalmente, de depósitos de descalcificación de materiales calizos. Además, el descenso de lluvia anual en la parte alta de la subcuenca Pajaroncillo (lluvia por el frente atlántico) está originando una disminución de la recarga al acuífero.

Tabla 3. Factores correctores calibrados en la subcuenca Pajaroncillo para un intervalo de tiempo diario. Periodo $1^{\circ}$ de Julio del 2002 al 31 de Julio del 2003

\section{Factores Correctores}

FC-1 Almacenamiento estático

FC-2 Evapotranspiración

0,636

FC-3 Infiltración

0,924

FC-4 Escorrentía directa

0,004

FC-5 Percolación

0,248

FC-6 Interflujo

FC-7 Pérdidas subterráneas

22 Flujo Base

501,774

0,000

2,389

FC-9 Velocidad en los cauces

0,834

Factor de interpolación de lluvia

0,000

La calibración y validación del modelo hidrológico generó buenos resultados, evaluándose el rendimiento del modelo a través del índice de Nash-Sutcliffe, el error en el volumen y el error cuadrático medio RMSE (Tabla 4).
Estos resultados óptimos se visualizan en las Figuras 6, 7, 8 y 9; el hidrograma del caudal simulado es muy cercano al del caudal observado. Las figuras permiten también observar el comportamiento de la lluvia media de la cuenca para los diferentes periodos, coincidiendo los caudales picos con las lluvias máximas.

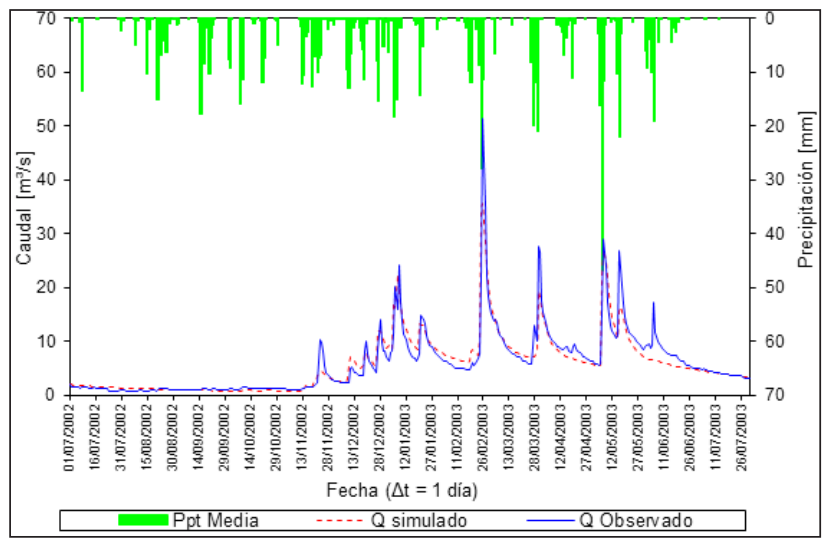

Figura 6. Hidrograma diario del caudal observado y simulado a la salida de la subcuenca Pajaroncillo después de la calibración. Periodo de análisis: $1^{\circ}$ Julio 2002 al 31 Julio 2003



Figura 7. Hidrograma diario del caudal observado y simulado a la salida de la subcuenca Pajaroncillo después de la validación temporal. Periodo de análisis: $1^{\circ}$ Marzo 2000 al 31 Octubre 2009

Tabla 4. Resultados de la calibración, validación temporal y validación espacio-temporal con el modelo TETIS para un intervalo de tiempo diario

\begin{tabular}{|c|c|c|c|c|}
\hline \multirow[b]{2}{*}{ Principales Características } & \multicolumn{2}{|c|}{ Pajaroncillo } & Alarcón & Contreras \\
\hline & Calibración & $\begin{array}{l}\text { Validación } \\
\text { Temporal }\end{array}$ & $\begin{array}{c}\text { Validación } \\
\text { Espacio-Temporal }\end{array}$ & $\begin{array}{c}\text { Validación } \\
\text { Espacio-Temporal }\end{array}$ \\
\hline & Jul02-Jul03 & Mar00-Oct09 & Feb02-Oct09 & Mar00-Oct09 \\
\hline Caudal máximo observado (m3/s) & 51,525 & 59,327 & 125,891 & 72,523 \\
\hline Caudal máximo simulado (m3/s) & 35,793 & 43,993 & 80,363 & 86,111 \\
\hline $\mathrm{N}^{\circ}$ de datos & 396 & 3511 & 2472 & 3505 \\
\hline Caudal medio observado & 5,964 & 3,391 & 13,801 & 5442 \\
\hline Volumen Observado (Hm3) & 204,067 & 1028,772 & 2947,645 & 1648,023 \\
\hline Volumen Simulado (Hm3) & 199,171 & 1129,975 & 2960,145 & 1658,434 \\
\hline Error en Volumen $(\%)$ & $-2,40$ & 984 & 0,42 & 0,63 \\
\hline Índice de Nash-Sutcliffe (NSE) & 0,874 & 0,810 & 0,509 & 0,620 \\
\hline $\begin{array}{l}\text { Error Cuadrático Medio (RMSE) } \\
(\mathrm{m} 3 / \mathrm{s})\end{array}$ & 2,070 & 1,916 & 9,872 & 3,927 \\
\hline
\end{tabular}


El SAIH obtiene por balance el caudal de entrada $\mathrm{I}(\mathrm{t})$ con datos medidos del caudal de salida $\mathrm{O}(\mathrm{t})$ y variación del nivel del embalse. Esta variación del nivel del embalse origina las oscilaciones (pelos) que se visualizan en el caudal diario observado (Figs. 8 y 9) y que están deteriorando los estadísticos obtenidos. Sin embargo, a nivel mensual, estas oscilaciones desaparecen ya que los valores extremos se compensan, mejorando los estadísticos, tal como se aprecia en las Figs. 10 y 11.



Figura 8. Hidrograma diario del caudal observado y simulado a la entrada del embalse Alarcón después de la validación espacio-temporal. Periodo de análisis: 1

Febrero 2002 al 31 Octubre 2009

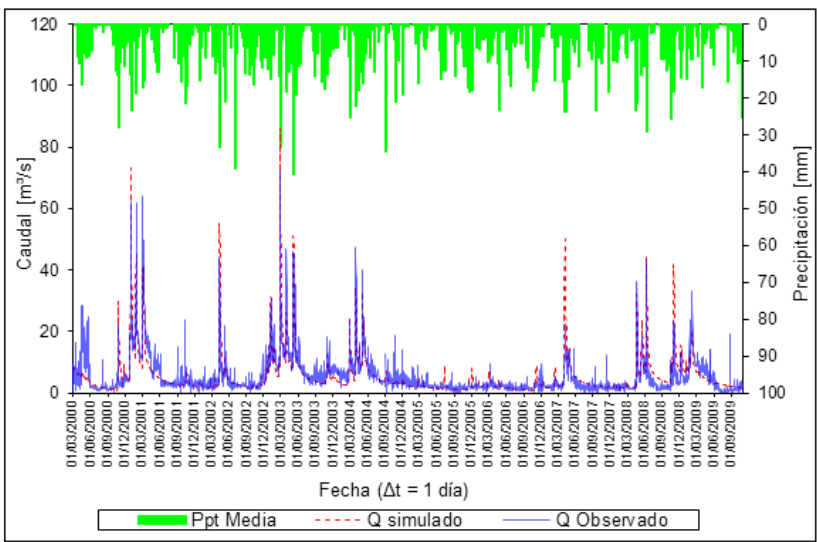

Figura 9. Hidrograma diario del caudal observado y simulado a la salida de la entrada del embalse Contreras de la validación espacio-temporal. Periodo de análisis: $1^{\circ}$ Marzo 2000 al 31 Octubre 2009

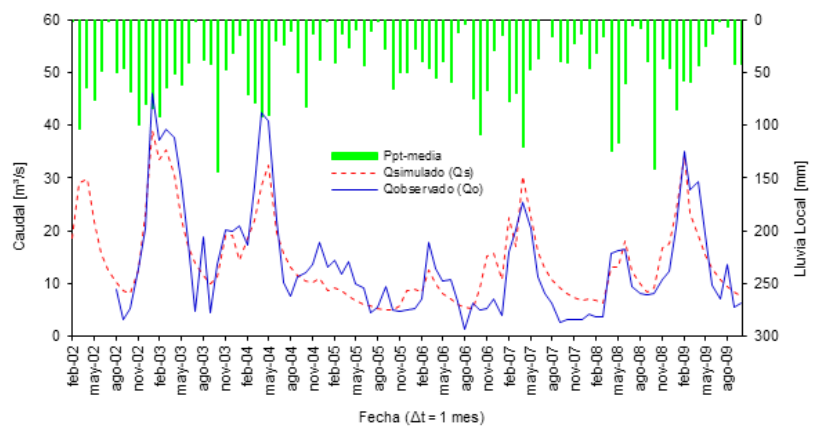

Figura 10. Hidrograma observado y simulado con validación espacio-temporal a la entrada del embalse Alarcón para un periodo de tiempo mensual. Periodo de análisis: Febrero 2002 a Octubre 2009

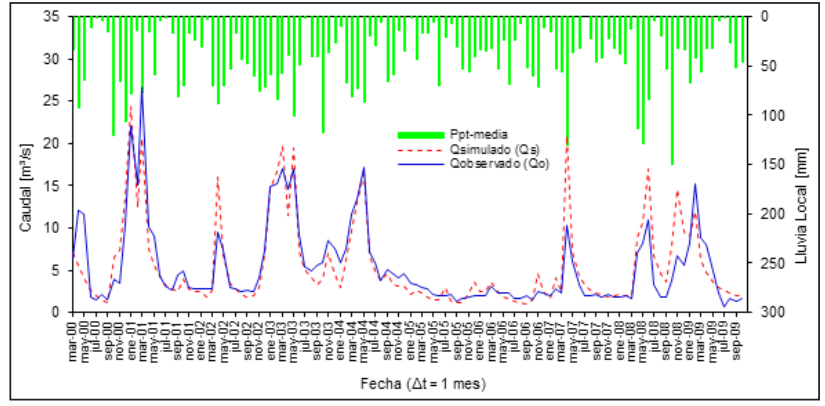

Figura 11. Hidrograma observado y simulado con validación espacio-temporal a la entrada del embalse Contreras para un periodo de tiempo mensual. Periodo de análisis: Marzo 2000 a Octubre 2009

La validación a la entrada del embalse Alarcón corresponde a un régimen natural, ya que en este proceso no ha influido el trasvase Tajo-Segura.

\section{Conclusiones}

La lluvia en la cuenca del Júcar es un proceso complejo que tiene asociado un grado de incertidumbre que se caracteriza por su enorme variabilidad temporal y espacial. En verano, lluvias intensas de corta duración, con picos aislados de valor muy alto, originadas por sistemas convectivos de mesoescala SCM típicos del Mediterráneo. En invierno, los valores más altos se concentran en la parte alta de la cuenca originados por la cadena de montañas del sistema ibérico. Ante esto, la modelación hidrológica en la cuenca del rio Júcar con el modelo TETIS tuvo óptimos resultados con índices de Nash-Sutcliffe de 0,87 (calibración), 0,81 (validación temporal) y 0,51-0,62 (validación espaciotemporal) por lo que puede proporcionar información que servirá de ayuda en diversas aplicaciones hidrológicas, gestión del agua y del medio ambiente en la cuenca del río Júcar y, con ello, fortalecer la capacidad de gestión de los recursos hídricos, mejorar la predicción del clima y desastres naturales y ofrecer rigor científico que ayude a tomar decisiones informadas.

\section{Agradecimientos}

El presente trabajo ha sido subvencionado por el Ministerio español de Ciencia e Innovación, a través del proyecto Consolider-Ingenio "SCARCE” (2010-CSD2009-00065).

\section{Literatura citada}

DIHMA. 2001. Un Modelo de Predicción de Crecidas para el SAIH de la Confederación Hidrográfica del Tajo. Informe Final para UTE SAIH TAJO.

DIHMA. 2002. Investigación conjunta sobre generación de mapas de caudales máximos en la cuenca del Júcar. Informe Final para el CEDEX.

Duan, Q.; Soroosh, S. y Gupta, H.V. 1992. Effective and efficient global optimization for conceptual rainfall-runoff models. Water Resources Research, 28 (4): 1015-1031.

Eckhardt, K. y Arnold, J. G. 2001. Automatic calibration of a distributed catchment model. Journal of Hydrology, 251, 103-109. 
FAO. 2006. Evapotranspiración del cultivo: Guias para la determinación de los requerimientos de agua de los cultivos. Estudio FAO. Riego y Drenaje 56. Roma.

Frances, F., Velez, J. y Velez, J.J. 2007. Split-parameter structure for the automatic calibration of distributed hydrological models. Journal of Hydrology, 332 (1-2): 226-240.

Frances, F.; Vélez, J.J.; Vélez, J.I. y Puricelli, M. 2002. Distributed modelling of large basins for a real time flood forecasting system in Spain. En Second Federal Interagency Hydrologic Modelling Conference. Las Vegas, USA.

Khakbaz, B.; Imam, B.; Hsu, K. y Soroosh, S. 2009. From lumped to distributed via semi-distributed via semidistributed: Calibration strategies for semi-distributed hydrologic models. Journal of Hydrology.

Vélez, J.I. 2001. Desarrollo de un modelo hidrológico conceptual y distribuido orientado a la simulación de las crecidas. (Ph.D.Thesis). Departamento de Ingeniería Hidráulica y Medio Ambiente, Universidad Politécnica de Valencia.

Vélez, J.I.; Vélez, J.J. y Frances, F. 2002. Modelo distribuido para la simulación hidrológica de crecidas en grandes cuencas. En XX Congreso Latinoamericano de Hidráulica. La Habana, Cuba.

Vélez, J.J. y Frances, F. 2005. Automatic calibration of initial state variables for flood forecasting using a distributed model. International conference on innovation advances and implementation of flood forecasting technology.

Vélez, J.J. y Frances, F. 2008. Calibración automática de las condiciones iniciales de humedad para mejorar la predicción de eventos de crecida. Avances en Recursos Hidráulicos, 18: 25-36.

Vélez, J.J. y Frances, F. 2009. Calibración automática de las condiciones iniciales de humedad para mejorar la predicción de eventos de crecidas. Avances Recursos Hidráulicos.

Vélez, J.J.; Frances, F. y López, F. 2007. Calibration strategy for hydrological distributed conceptual models. IUGG XXIV General Assembly, "Earth: our changing planet”, IAHS-PUB session HW2005: From Measurements and Calibration to Understanding and Predictions. 